\title{
A Review of Quantum Games
}

\author{
Gaon $\mathrm{Kim}^{1 *}$ and Eung-won $\mathrm{Nho}^{2}$
}

Over the past two decades, the quantum mechanical concepts of superposition and entanglement have been applied in game theory to produce novel and interesting results. Quantization offers significant improvements to classical games that cannot be realized using purely classical strategy spaces. Because quantum game theory is a recent development with both merits and limitations, this review attempts to critically evaluate existing research as well as gaps in the literature requiring further research. The literature is classified into four categories of games based on differences in quantization schemes and results: quantum simultaneous non-zero-sum games, quantum simultaneous zero-sum games, quantum coalitional games and quantum sequential games. The first two categories exhibit the results of Pareto efficiency and improved payoffs, but the literature reviewed does not sufficiently analyze the role of strategic coordination in bringing about such improvements. Quantum coalitional games also have improvements over their classical counterparts, often leading to cores that yield higher payoffs to a greater number of players, given a quantization scheme that encompasses all players' strategy spaces. However, the mechanism through which these improvements are realized is generally unclear. Finally, quantum sequential games exhibit cooperative behavior among players that is absent in the corresponding classical games. This review concludes that quantum games have significant advantages over their classical counterparts and suggests the role of strategic coordination in quantum games as a fruitful direction for future research.

\section{INTRODUCTION}

It is perhaps not a coincidence that the mathematician John von Neumann was a significant contributor in both economic game theory and quantum mechanics (von Neumann, 1953; von Neumann, 1955). Although classified as two discrete subjects of modern science, game theory and quantum mechanics exhibit deep, interesting connections (Meyer, 1999). At a glance, the two topics seem completely unrelated indeed: game theory is an analytical tool used to model and study the decision-making process of rational economic entities, whereas quantum mechanics is a branch of physics describing the nature of motion at the smallest scales of physical quantities. However, over the past two decades, numerous surprising connections have been found linking the fields after two key concepts from quantum mechanics - superposition and entanglement - were applied in classical game theory. Superposition refers to the property that a physical state can be a sum of multiple distinct states, each of which has a probability of being observed; entanglement is a phenomenon in which multiple particles are related, such that each of their quantum states is dependent on each other, regardless of physical distance (Nielsen and Chuang, 2000). A classical game is quantized when (i) the outcomes of the game are entangled and superposed, (ii) players' strategy sets are

${ }^{1}$ K. International School Tokyo, 1-5-15 Shirakawa, Koto-ku, Tokyo, Japan

2 Department of Economics, Chungnam National University, 99 Daehak-ro, Gung-dong, Yuseong-gu, Daejon, South Korea

*To whom correspondence should be addressed: gaonkim7@gmail.com

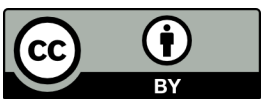

Except where otherwise noted, this work is licensed under https://creativecommons.org/licenses/by/4.0

doi:10.22186/jyi.37.2.10-16 expanded to include unitary operators (termed "quantum strategies"), or (iii) both of the above occur. These quantum mechanical applications in games produce various novel and interesting results. In this review, the term "novel" refers to a result in quantum game theory that is favorable and could not be realized in classical game theory. Quantum game theory literature focuses on a number of such results to establish the topic's significance.

Pareto efficiency refers to an outcome in a game such that there are no other possible outcomes that give higher payoffs to a non-zero number of players without decreasing any player's payoff. Conversely, Pareto inefficiency is observed when the Nash equilibrium of the game - an outcome where all players of a game do not have an incentive to change their strategies - does not exhibit Pareto efficiency (Nash, 1951). Games that are Pareto inefficient under the classical paradigm can be made efficient through the use of quantization, which is a novel result beneficial to the players (Eisert, Wilkens, and Lewenstein, 1999). Another key result is higher payoffs for players in the game, which directly indicates that they have benefitted from quantization (Meyer, 1999). Quantization can also lead to new coalitions among players in the game that gives higher payoffs to a greater number of players (Chen, Hogg, and Beausoleil, 2002). These results, obtained uniquely in quantum games, are significant as they expose a deep and rich econophysical connection wherein quantization is conducive to strategic coordination. Although the current lack of quantum technology imposes significant limitations on immediate practical applications of the theory, the accuracy of a few simple quantum games has been supported with experimental evidence through computer science, suggesting the potential relevance of the theory once necessary technological advances are made (Du et al., 2002; Prevedel et al., 2007; Schmid et al., 2010).

Research conducted on quantum game theory may be clas- 
sified into four categories: quantum simultaneous non-zero-sum games, quantum simultaneous zero-sum games, quantum coalitional games, and quantum sequential games. These games are fundamentally different, as simultaneous games have players choose their strategies independently, whereas players compete as groups in coalitional games and players take turns choosing their strategies in sequential games (with at least some information on others' strategies in previous turns). This review attempts to examine research from the four major categories described and discuss the significances of their results, as well as gaps and limitations in the current theory. Based on comparisons between classical and quantum games, this review asserts that quantization is significant in allowing for strategic coordination between players that improve payoffs, leading to novel results favorable for the game's players.

\section{QUANTIZATION SCHEME FOR SIMULTANEOUS NON- ZERO-SUM STRATEGIC GAMES}

The quantization scheme used prevalently in simultaneous nonzero-sum strategic games was proposed by Eisert et al. (1999). It is widely researched using the Quantum Prisoner's Dilemma (QPD) as well as the Quantum Battle of Sexes (QBoS). Eisert et al.'s presentation of the quantization scheme through the QPD is outlined below and shows a remarkable example of quantum game theory - namely, the emergence of a Pareto efficient Nash equilibrium benefitting both players. However, further research is needed to investigate the role of strategic coordination in the emergence of this favorable outcome.

Classical Prisoner's Dilemma (PD) is a standard game that reveals how two rational players, Alice (player $i=1$ ) and Bob (player $i=2$ ), may choose strategies that lead to an outcome that is not in their best interest - a Pareto inefficient outcome. In the PD, Alice and Bob are accused of a crime, and both players can adopt one of two possible strategies - confessing to the crime or defecting from doing so. Each possible pair of strategies chosen by Alice and Bob lead to a particular payoff for each player, enlisted in the matrix of Table 1. It is clear from this payoff matrix that "defect" is the dominant strategy for both players, hence the Nash equilibrium $(\hat{D}, \hat{D})$. However, because both players can improve their respective payoffs by shifting to "confess" in $(\hat{C}, \hat{C})$, the equilibrium is a Pareto inefficient outcome. Thus, the players are placed in a "dilemma".

The QPD builds on the classical PD by using quantization to produce an efficient outcome. Firstly, the strategy space for both

Table 1. The playoff matrix for Classical Prisoner's Dilemma (PD; Eisert et al., 1999). The players face a "dilemma" as the Nash equilibrium of $\mathrm{O}$ is Pareto inefficient.

\begin{tabular}{|c|c|c|c|}
\hline \multicolumn{2}{|c|}{} & \multicolumn{2}{|c|}{ Bob } \\
\cline { 3 - 4 } \multicolumn{2}{|c|}{} & $\hat{\boldsymbol{C}}$ & $\hat{\boldsymbol{D}}$ \\
\hline \multirow{3}{*}{ Alice } & $\hat{\boldsymbol{C}}$ & 3,3 & 0,5 \\
\cline { 2 - 4 } & $\hat{\boldsymbol{D}}$ & 5,0 & 0,0 \\
\hline
\end{tabular}

players is expanded so that it is quantized:

$$
A(\theta, \varphi)=\left(\begin{array}{cc}
e^{i \varphi} \cos \theta / 2 & \sin \theta / 2 \\
\sin \theta / 2 & e^{-i \varphi} \cos \theta / 2
\end{array}\right)
$$

where $\theta \in[0, \pi]$ and $\varphi \in[0, \pi / 2]$ (Eisert et al., 1999). This unitary strategy space encompasses classical strategies: "confess" is represented by the operator $\hat{C}=\left(\begin{array}{ll}1 & 0 \\ 0 & 1\end{array}\right)$ and "defect" by $\hat{D}=$ $\left(\begin{array}{cc}0 & 1 \\ -1 & 0\end{array}\right)$

Another feature is introduced in the QPD: the final state of the game determines the payoff. The initial state is defined as

$$
\left|\psi_{i}\right\rangle=\hat{J}|C C\rangle
$$

and the final state of the game, determined according to the two players' strategies $A_{i=1}$ and $A_{i=2}$, is

$$
|\psi f\rangle=\hat{J}\left(A_{i=1} \otimes A i_{=2}\right) \hat{J}|C C\rangle
$$

where $|C\rangle=\left(\begin{array}{l}1 \\ 0\end{array}\right)$ and $|D\rangle=\left(\begin{array}{l}0 \\ 1\end{array}\right)$; the unitary operator $\hat{J}$ determines the extent of entanglement present in the state before Alice and Bob apply their strategies. Mathematically, an entanglement of two states "0" and " 1 ", represented by vectors $|0\rangle$ and $|1\rangle$, is denoted by $|01\rangle$, which is the tensor product $|0\rangle \otimes|1\rangle$, indicating that the two states are observed together. In the case of maximal entanglement that is examined, $\left|\psi_{i}\right\rangle=\frac{1}{\sqrt{2}}(|C C\rangle+i|D D\rangle)$. Since the strategy space is unitary, the final state has the magnitude of 1 as $\left|\psi_{i}\right\rangle$ and is a superposition of the entangled orthonormal basis $|C C\rangle,|D C\rangle,|C D\rangle$, and $|D D\rangle$, each of which represents one of the classical outcomes of the game (i.e. $\left|\psi_{f}\right\rangle=\frac{1}{\sqrt{2}}(|C C\rangle+|C D\rangle)$ gives payoff $\frac{1}{2}(3,3)+\frac{1}{2}(0,5)=$ $(1.5,4)$ as it represents the case where Alice confesses and Bob confesses with probability $1 / 2$, while Alice confesses and Bob defects with probability $\frac{1}{2}$ ). Note that $|C\rangle$ and $|D\rangle$ represent

"confess" and "defect" within the context of the final state, but are not actual strategies open to players - these vectors have been introduced simply as a way of determining players' payoffs using the outcomes of the classical PD.

The case of maximal entanglement caused by the operator $\hat{J}$ is significant due to its unique property of containing a Pareto efficient Nash equilibrium when both players choose the strategy $\hat{Q}=A(0, \pi / 2)=\left(\begin{array}{cc}i & 0 \\ 0 & -i\end{array}\right)$ (Eisert et al., 1999). In other words, the "dilemma" present in PD of an inefficient equilib- 
Table 2. The payoff matrix for Quantum Prisoner's Dilemma (QPD; Grabbe, 2005). The "dilemma" of PD is solved as $(\hat{Q}, \hat{Q})$ is a Nash equilibrium exhibiting Pareto efficiency.

\begin{tabular}{|c|c|c|c|c|}
\hline \multicolumn{2}{|c|}{} & \multicolumn{3}{|c|}{ Bob } \\
\cline { 3 - 5 } \multicolumn{2}{|c|}{} & $\hat{\boldsymbol{C}}$ & $\hat{\boldsymbol{D}}$ & $\hat{\boldsymbol{Q}}$ \\
\hline \multirow{3}{*}{ Alice } & $\hat{\boldsymbol{C}}$ & 3,3 & 0,5 & 1,1 \\
\cline { 2 - 5 } & $\hat{\boldsymbol{D}}$ & 5,0 & 0,0 & 0,5 \\
\cline { 2 - 5 } & $\hat{\boldsymbol{Q}}$ & 1,1 & 5,0 & 3,3 \\
\hline
\end{tabular}

rium has been solved as $\hat{Q}$ gives rise to an efficient equilibrium (Eisert et al., 1999). As shown in the matrix of Table 2, the equilibrium $(\hat{Q}, \hat{Q})$ gives payoffs of $(3,3)$ equivalent to that of $(\hat{C}$, $\hat{C})$ in the classical game, which was previously seen to be Pareto efficient but not an equilibrium in PD. Thus, the creation of an efficient equilibrium following quantization is a novel result. Note that Table 2 does not contain the entire set of strategies and payoffs, which are continuous and would result in a multidimensional graph rather than the simple and discrete matrix presented; this method of presentation is motivated by Grabbe (2005).

The question then arises: how has the quantization of the PD resulted in this efficiency? Considering the final state interpretation of the game, it is clear that quantization of the game has allowed for coordination between Alice and Bob's classical strategies of $|C\rangle$ and $|D\rangle$, as they were entangled. In fact, it is because of this strategic coordination in the final state that Alice and Bob attain an efficient equilibrium equivalent to both players confessing. However, one cannot conclude from this strategic coordination that the QPD is not a simultaneous game, which by definition requires independence in players' choice of strategies, because the quantum strategies themselves are indeed chosen independently from $\mathrm{A}(\theta, \varphi)$ - the final state interpretation is simply a way of determining the players' payoff using the outcomes of the classical game. This aspect of strategic coordination due to quantization is significant in analyzing quantum games but has not been discussed in the literature reviewed regarding simultaneous non-zero-sum strategic games. Research on this area of strategic coordination would further understanding of how quantization leads to improvements over classical games. Although Eisert et al. (1999) established that quantization of games does lead to certain favorable outcomes of cooperation, the mechanism through which quantization leads to improvements is unclear. There is evidence that strategic coordination due to quantization is involved in this cooperation, so investigating the mechanisms through which quantization causes strategic coordination is likely to offer insight into this mechanism.

Another aspect of the QPD that could benefit from further research is the role of the operator $\hat{J}$ in the quantization scheme outlined above. It seems that $\hat{J}$ is inconsistent with the original context of the PD, which involves two players suspected of a crime strategically trying to benefit themselves (Enk and Pike, 2002). In the QPD, $\hat{J}$ is helpful to the players, who are prisoners, as it creates entanglement, leading to an efficient equilibrium. However,
Table 3. The payoff matrix for Classical Battle of Sexes (BoS; Marinatto $\&$ Weber, 2000). There exists a problem of coordination of both $(\hat{O}, \hat{O})$ and $(\hat{T}, \hat{T})$ are Nash equilibria.

\begin{tabular}{|l|c|c|c|}
\hline \multicolumn{2}{|c|}{} & \multicolumn{2}{c|}{ Bob } \\
\cline { 3 - 4 } \multicolumn{2}{|c|}{} & $\hat{\boldsymbol{O}}$ & $\hat{\boldsymbol{T}}$ \\
\hline \multirow{3}{*}{ Alice } & $\hat{\boldsymbol{O}}$ & 3,3 & 0,5 \\
\cline { 2 - 4 } & $\hat{\boldsymbol{T}}$ & 5,0 & 0,0 \\
\cline { 2 - 4 } & \multicolumn{3}{|c|}{$\alpha>\beta>\gamma$} \\
\hline
\end{tabular}

considering the context of the original game, it is unclear what entity could play the role of $\hat{J}$, allowing for the prisoners' strategic coordination through entanglement. A possibility might be the prisoners' interrogator or attorney, but such a scenario is unrealistic because neither have any incentive to help the prisoners (Enk and Pike, 2002). Enk and Pike's article suggests that it is necessary to carefully define the entity playing the role of $\hat{J}$ in a way consistent with the original context and that further research is required.

One may follow Enk and Pike's line of reasoning and question the significance of the strategy space given in Equation 1, given its arbitrary appearance. It is hard to find a meaningful interpretation to defining the strategy space in this particular way (Benjamin and Hayden, 2001). On the other hand, a more physically "natural" way of defining the strategy space would include all possible unitary operators that do not affect entanglement, reflecting an interpretation in which the players can adopt anything that is possible within quantum mechanics (Benjamin and Hayden, 2001). In fact, Benjamin and Hayden (2001) found that no equilibrium of pure strategies exists using this strategy space for the QPD. However, despite this limitation, Eisert et al.'s restriction in strategy space as given in Equation 1 is significant and necessary precisely because it can produce an efficient equilibrium in all two-player simultaneous games, not just for the QPD (Du et al., 2000). Thus, although Benjamin and Hayden (2001) correctly point out that Eisert et al.'s quantization scheme restricts the strategy space without a realistic physical interpretation, this restriction is necessary in order to obtain the desired result of an efficient equilibrium in any two-player simultaneous game (Du et al., 2000).

Another example of a quantum non-zero-sum simultaneous strategic game is the QBoS, first proposed and studied by Marinatto and Weber (2000). The payoff matrix for the Classical Battle of Sexes (BoS) is shown in Table 3. The game is played between Alice (player $i=1$ ) and Bob (player $i=2$ ), who both have two strategies $\hat{O}$ - going to the opera - and $\hat{T}-$ watching television. Both $(\hat{O}, \hat{O})$ and $(\hat{T}, \hat{T})$ are Nash equilibria in this game and give payoffs of $\alpha$ to one player and $\beta$ to another. However, if they fail to choose the same strategy - that is, if they choose either $(\hat{O}, \hat{T})$ or $(\hat{T}, \hat{O})$ - the players will receive the worst possible payoff of $(\gamma, \gamma)$. Thus, the classical BoS is a game of coordination for the players to choose the same strategy. The QBoS is also played by Alice (player $i=1$ ) and Bob (player $i=2$ ), and the same quantization scheme developed by Eisert et al. is used (Benjamin, 2000; 
Marinatto and Weber, 2000), but the strategy space is extended to the set of all two-by-two unitary matrices. The classical strategies in the strategy set are represented by the operators (i) $\hat{O}=\left(\begin{array}{ll}1 & 0 \\ 0 & 1\end{array}\right)$ and (ii) $\hat{T}=\left(\begin{array}{cc}0 & 1 \\ -1 & 0\end{array}\right)$. The final state is therefore a superposition of the orthonormal basis $|O O\rangle,|O T\rangle,|T O\rangle$, and $|T T\rangle$ such that $|O\rangle=\left(\begin{array}{l}1 \\ 0\end{array}\right)$ and $|T\rangle=\left(\begin{array}{l}0 \\ 1\end{array}\right)$. Marinatto and Weber (2000) proved through algebraic reasoning that there exists a certain pairing of mixed quantum strategies between Alice and Bob such that $\left|\psi_{f}\right\rangle=\frac{1}{\sqrt{2}}(|O O\rangle+|T T\rangle)$. Consequently, the expected payoff to both players is $(\alpha+\beta) / 2$, which can be interpreted as the average payoff of the two Nash equilibria $(\hat{O}, \hat{O})$ and $(\hat{T}, \hat{T})$ (Marinatto and Weber, 2000). This is observed to be an improvement over the classical BoS (Marinatto and Weber, 2000). Similar to the QPD, this improvement seems to be the result of strategic coordination between Alice and Bob due to quantization. Because the two players' strategies are entangled, they can readily be coordinated to obtain the desirable outcomes of either $|O O\rangle$ or $|T T\rangle$, where the players choose the same strategies.

As noted by Benjamin (2000), one limitation of the QBoS is that the game requires mixed quantum strategies to obtain the desired equilibrium, yielding yet another uncertainty in strategies. Mixed strategies, whether quantum or classical, are probabilistically determined, so both desirable and undesirable outcomes can arise - the expected payoff simply gives the average of the different possible outcomes weighted according to their probabilities. In fact, a similar result of an equilibrium can be obtained even in the classical version if mixed classical strategies are used (Grabbe, 2005). When using mixed classical strategies in the BoS, the players can decide on a probability distribution between the two strategies $\hat{O}$ and $\hat{T}$ that will maximize their respective expected payoffs. Defining $p *$ as the probability of Alice choosing $\hat{O}$ and $q *$ as the probability of Bob choosing $\hat{O}$, the expected payoffs for Alice and Bob can be optimized using calculus. This computation yields an expected payoff of $\pi=\frac{\alpha \beta-\gamma^{2}}{\alpha+\beta-2 \gamma}$ to both players, which is

lower than the expected payoff of $\frac{\alpha+\beta}{2}$ for the QBoS (Marinatto and Weber, 2000). Thus, although the QBoS does result in higher expected payoffs than the BoS due to coordination through quantization, since the QBoS requires mixed strategies to obtain its equilibrium, which is possible even in the classical version, the advantage of the QBoS over the BoS does seem to be undermined.

\section{QUANTIZATION SCHEME FOR ZERO-SUM SIMULTA- NEOUS STRATEGIC GAMES}

Because Eisert et al.'s quantization scheme uses both quantum strategies and entanglement to quantize a game, considering the effects of using only quantum strategies without entanglement may offer insight into their unique advantages (Du et al., 2000). Meyer's scheme, which does not use the operator $\hat{J}$, exposes the advantages of quantum strategies by comparing them with classical strategies without entanglement (Meyer, 1999; Du et al., 2000). Despite the absence of entanglement in Meyer's scheme, the results of the zero-sum quantum game are highly analogous to those of Eisert et al.'s scheme in which payoffs are improved through quantization. Meyer's scheme is outlined below.

Meyer's quantum game is equivalent to the classical "Matching Pennies" game in all respects other than its strategy set, which has been expanded into the entire set of unitary two-by-two operators. A penny is placed head up initially. Then, without looking at the penny each time, player $i=1$, player $i=2$, and player $i=$ 1 (once again) apply strategies of their choice to the penny in the order listed. If the penny appears as "head" in the end, player $i=1$ and $i=2$ receive payoffs of +1 and -1 respectively, and vice versa in the case of "tail". Thus, the game is classified as zero-sum. Though the game is played in sequence, it is still a simultaneous game because neither players receives any information about his opponent's strategy nor the state of the penny throughout the game. In the classical game, both players are restricted to the strategy set of "flip" and "not flip," represented by $\hat{F}=\left(\begin{array}{ll}0 & 1 \\ 1 & 0\end{array}\right)$ and $\hat{N}=\left(\begin{array}{ll}1 & 0 \\ 0 & 1\end{array}\right)$

respectively, which may be mixed probabilistically. However, in Meyer's quantum game, player $i=1$ alone has access to quantum strategies in the form of the unitary operator $A(a, b)$ $=\left(\begin{array}{cc}a & b \\ \bar{b} & -\bar{a}\end{array}\right)$. The state of the game, which is a superposition of "head" and "tail," is represented by a linear combination of $|H\rangle=\left(\begin{array}{l}1 \\ 0\end{array}\right)$ and $|T\rangle=\left(\begin{array}{l}0 \\ 1\end{array}\right)$. Quantum strategies induce

the final state of the game to be in the form $\left|\psi_{f}\right\rangle=a|H\rangle+b|T\rangle$ , which is superposed, but not entangled. In general, the initial state of the game is $|H\rangle$, and ultimately

$$
\left|\psi_{f}\right\rangle=A_{2}\{p F+(1-p) N\} A_{l}|H\rangle
$$

where $A_{1}$ and $A_{2}$ are player $i=1$ 's strategies in chronological order, and $p$ is the probability at which player $i=2$ will choose "flip" as his strategy. Meyer (1999) computed the final state density matrix - a matrix showing the respective probability amplitudes of each of the possible states a superposed state may be in - to assert that if $A_{1}=A_{2}=A\left(\frac{1}{\sqrt{2}}, \frac{1}{\sqrt{2}}\right)$, the final state will be "head", independent of player $i=2$ 's choice of $p$. The Nash equilibrium thus gives payoffs of +1 to the quantum player and -1 to the classical player. This contrasts with the classical equilibrium of the Matching Pennies game, which gives an expected payoff of zero to both players (Meyer, 1999). This analysis of Matching Pennies game therefore suggests that a simultaneous zero-sum game be- 
tween a quantum player and a classical player will be advantageous for the former. Meyer (1999) generalized this observation to prove that (i) all simultaneous zero-sum games do have a Nash equilibrium when played between a quantum player and classical player, and (ii) the expected payoff for the quantum player in this equilibrium is at least as great as the expected payoff when he plays mixed classical strategies like the other player (Meyer, 1999). In other words, the observation that the quantum player has an advantage over the classical player holds in general for simultaneous zero-sum games. Using quantization in this way is another novel result that can be utilized to improve players' payoffs.

The observation above has been verified through other games as well. The quantized "Card Game "presented by Du et al. (2000) is an interesting example: the initial zero-sum game is between a quantum player and a classical player using mixed strategies, each with zero expected payoff. However, consistent with the theorem above, when the quantum player is reverted into a classical player by restricting his initially quantum strategy space to simply mixed classical strategies, he actually receives less payoff than his opponent. It is evident from the quantized Card Game that the quantum player did possess an advantage when allowed to use quantum strategies.

This result of Meyer's quantization scheme implies that quantum strategies have properties absent in classical ones that can make the quantization of a classical game an advantageous choice. The invariance of the final state suggests that quantum strategies create strategic links between the players. When player $i=2$ chooses to flip the penny, his opponent also ends up flipping the penny. However, when he chooses not to flip, his opponent also ends up not flipping the penny. This dependency between strategies from the perspective of the final state is a consequence of the extension of the strategy space to the quantum domain. The player capable of playing with the quantum strategies is at an advantage because he can manipulate the strategic link to benefit himself. Consequently, quantum strategies without entanglement can create strategic links in accordance with the final state between players in ways analogous to the QPD. This is a significant observation regarding the impact quantization of strategies has on a classical game without entanglement.

\section{Quantum Coalitional Games}

The effects of quantization observed in various simultaneous games can be readily applied in coalitional games. Some methods illustrated in this section that are used to quantize coalitional games include sharing entangled states, using quantum strategies, and entangling players' states with others. The appropriate quantization of coalitional games yields novel, significant results of effective cooperation between players resulting from strategic coordination, often leading to win-win situations, as explored in games such as the Public Goods Game and the Minority Game. A wider variety of quantum coalitional games must nonetheless be researched using a greater number of methods to determine the general mechanism through which quantization leads to these improved outcomes.

An illuminating example, motivated by the idea of multiplayer entanglement, is Mermin's Multiplayer Pseudo-Telepathy
Game between $n$ players (Brassard et al., 2004; Mermin, 1990). A review of pseudo-telepathy games in general is provided by Brassard, Broadbent, and Tapp (2005). In the formulation of Mermin's Multiplayer Pseudo-Telepathy Game by Brassard et al. (2004), a single state $x_{i}$ is given to each player who is then allowed to apply a transformation $T_{i}$ to get $T_{i}: x_{i} \rightarrow y_{i}$. Given that $\sum_{i} x_{i}$ is even, all players receive a total payoff of +1 if and only if the following condition is satisfied:

$$
\frac{1}{2} \sum_{i} x_{i}=\sum_{i} y_{i}
$$

In all other cases, all players receive a payoff of -1 . The key aspect of this coalitional game is strategic coordination and cooperation among players, which is unachievable in the classical context as each player does not have perfect information about the strategies of all other players, preventing coordination. However, Brassard et al. (2004) present a proof for the existence of a set of transformations that results in the group certainly receiving a payoff of +1 . The desired set of transformations involves the sharing of an $n$-qubit, which is an entanglement of $n$ states of either $|0\rangle$, $|1\rangle$, or both (i.e. $\left.0^{3}\right\rangle=|0\rangle \otimes|0\rangle \otimes|0\rangle$ is an example of a 3 -qubit). The players first agree to share an entangled $n$-qubit state $\left.\frac{1}{\sqrt{2}}\left(\left|0^{n}+\right| 1^{n}\right\rangle\right)$ and to apply different operators on their parts of the shared $n$-qubit in accordance with the parity of their own given state $x_{i}$. This gives rise to a transformation that makes $y_{i}$ a superposition of only odd or only even states that satisfies Equation 5. In other words, quantization is used in these transformations to (i) create coordination between players' strategies through a shared entangled state and (ii) exploit quantum strategies to create a superposition of states with the same parity. In effect, superposition and the sharing of the n-qubit has led to the novel result of a winwin cooperation between the players, which was previously impossible in the classical context. Grabbe (2005) commented that the remarkable feature of this coalitional game is that, as a consequence of quantization, the core is achieved only when full cooperation occurs in the form of a grand coalition involving all players. Mermin's "Multiplayer Pseudo-Telepathy Game” suggests that the appropriate application of quantum mechanical concepts in coalitional games can result in the emergence of a core that is beneficial to a greater number of players by creating cooperation between players.

The "Public Goods Game" is a well-established game in standard economic theory and is also amenable to quantization. Chen et al. (2002) investigated a variation of the "Public Goods Game" in which there are $n$ players each indexed as $k=1, \ldots, n$. Player $k$ has an initial personal endowment of $y_{k}$ and contributes the amount $c_{k}$ to the public good. It is assumed that $y_{k}=1 \forall k$. Then, according to the production function $g(C)=a C / n$, where $C=\sum_{k} c_{k}$, the amount of public goods produced $x$ is determined. The utility of player $k$ is then determined through the function $Q_{k}(x, y)=x+y$ , where $y=y_{k}-c_{k}$ is the amount of private wealth remaining af- 
ter contributing to the public good. In this classical version, players will contribute nothing when $a<1$ and everything when $n<$ $a$, which are both Pareto efficient equilibria (Chen et al., 2002). However, when $1<a<n$, the equilibrium is for players to contribute nothing, which is an inefficient outcome; a coalition between all players to contribute everything would be Pareto efficient, but players have an incentive to cheat and contribute nothing for personal gains (Chen et al., 2002). The quantum version, also proposed by Chen et al. (2002), assigns the vector $|0\rangle=\left(\begin{array}{l}1 \\ 0\end{array}\right)$ to "cooperation" $\left(c_{k}=y_{k}=1\right)$ and $|1\rangle=\left(\begin{array}{l}0 \\ 1\end{array}\right)$ to "defection" $\left(c_{k}=0\right)$. Each player may choose a superposition of these vectors as their strategy. Chen et al. (2002) investigated three cases of entangling the players' strategies: (i) full entanglement between all players, (ii) entanglement between all possible pairs of players, and (iii) entanglement between neighboring pairs of players. All three cases are shown to have an equilibrium in which all players contribute between 0 and 1 in the equilibrium under $1<a<n$, which is not yet Pareto efficient, but still an improvement over the classical version (Chen et al., 2002). Once again, quantization of a classical game has created strategic coordination, which for the "Public Goods Game" resulted in a set of payoffs beneficial for all players. The "Quantum Public Goods Game" further demonstrates that coalitional games can exhibit cooperative behavior in the interest of all players through appropriate quantization. Furthermore, the quantization scheme presented seems to produce a valid version of the classical game that retains its coalitional nature - in both versions, players have a choice between cooperating with and defecting the coalition, with quantization as an effective reinforcement of the coalition.

More quantum coalitional games need to be quantized, as there is yet a limited number of quantized versions of such games, hindering the advancement of more accurate and general observations on these games. Various patterns and schemes of quantization are observed throughout the literature on this topic; in fact, the Public Goods Game has three different quantization schemes that each produces a different result (Chen et al., 2002). Additionally, some classical games such as the "Minority Game" produce unexpected results under quantization: while the classical Minority Game (players choose between two options, and those in the minority win) is primarily studied using an odd number of players to avoid balance of players, the quantum version can be designed to avoid the balance as much as possible, resulting in the same set of payoffs as the classical game for an odd number of players and higher payoffs for an even number of players (Benjamin and Hayden, 2001). The roles quantization and strategic coordination have in such a result is interesting but also bizarre. As with the "Public Goods Game," there are multiple ways to quantize the "Minority Game," but another bizarre result is that entanglement between pairs does not improve the payoffs in the Minority Game, which is inconsistent with the "Quantum Public Goods Game"
(Flitney and Hollenberg, 2008). More coalitional games must be quantized with various schemes and methodologies in order to further investigate the precise rules and mechanisms through which strategic coordination occurs and novel results are produced.

\section{QUANTUM SEQUENTIAL GAMES}

Sequential games also seem similarly affected by quantization. One such example is the quantum version of the quantity leadership model of a duopoly, also known as the Stackelberg model (Iqbal and Toor, 2002). The Stackelberg duopoly is a classical sequential game that attributes more profit to the leader firm, but profits are shared more equally in the form of a Cournot equilibrium - where no firm is the leader - when the game is quantized appropriately (Iqbal and Toor, 2002). Iqbal and Toor (2002) transformed the classical game into a quantum one by (i) using the quantization scheme equivalent to the $\mathrm{QBoS}$, but with a sequential structure and (ii) defining a function (Equation 6) that assigns each choice of quantity $q$ in the classical model to a unique value $x$ in the range $(0,-1]$ that describes the probabilistic distribution of the player's mixed quantum strategy. Iqbal and Toor (2002) then proved that their quantization scheme for the Stackelberg model leads to an outcome equivalent to that of a Cournot equilibrium, such that the leader firm has lost its dominance through quantization.

$$
x=\frac{1}{1+q}
$$

Although Iqbal and Toor (2002) concluded that the result is "counter-intuitive," the results seem reasonable and consistent with the effects of quantization observed throughout this review. In the classical version, the strategic link between the two firms works to the advantage of the leader firm, which has more market power over the other firm, whose strategy is in turn limited to "following" the leader. Quantization, on the other hand, strengthens the mutual strategic link between the two firms and allows the follower firm to have a greater and more direct impact on its competitor. Because the classical Cournot equilibrium does not contain a leader firm, the quantized Stackelberg equilibrium obtains an equivalent outcome as a result of this mutual and hence "fair" strategic link between the firms. This "fair" distribution of the firms' profits in the quantum Stackelberg equilibrium has not been observed in the classical version of the game, and is hence another example of a novel outcome induced through quantization.

As additional evidence, Li et al. (2002) followed Iqbal and Toor's investigation and found that the quantum version of the Cournot equilibrium - which is not a sequential game, but still an illustrative example - also exhibits more cooperative behavior than the classical version, in which firms act selfishly for their respective profits. The firms of the quantum Cournot duopoly exhibit a greater degree of cooperation than those in the classical Cournot duopoly in the sense that they choose strategies that effectively maximize their combined profits rather than their individual profits (Li et al., 2002). This behavior was observed to be increasingly strong for higher degrees of entanglement (Li et al., 
2002). The cooperative behavior between firms in a Cournot duopoly following the entanglement of their strategies is another novel result that is not observed in the classical version of the game, where firms simply maximize individual profits without regard for combined profits. The overall application of quantum mechanical concepts in sequential games seems to create strategic links that lead to novel outcomes of cooperative behavior and "fair" results.

\section{DISCUSSION}

This review demonstrates that the quantum mechanical concepts of entanglement and superposition are readily applicable to game theory, producing novel results that can be employed to better players' payoffs in a wide range of games. These results are not only absent in the original classical games, but also interesting because they pose a solution to the problems of these classical games, ultimately leading to beneficial results for players. For instance, the classical PD highlights the issue of an inefficient equilibrium dominating the efficient outcome; this problem is solved by quantization as superposition and entanglement lead to strategic links conducive to cooperation (Eisert et al., 1999). Similarly, the problem of coordination between players is solved in the BoS as quantization leads to a payoff where the two players choose the same strategy simultaneously (Marinatto and Weber, 2000). These solutions to the problems highlighted in classical games are found in zero-sum games, coalitional games and sequential games (Brassard et al., 2004; Chen et al., 2002; Iqbal and Toor, 2000; Meyer, 1999). Using quantization in these ways to solve issues of cooperation and coordination is the key idea of quantum game theory.

A fruitful direction for future research in quantum game theory is the role of strategic coordination in quantum simultaneous games. It was observed through the QPD and the QBoS that the final outcome of the quantum game suggests the existence of strategic coordination through quantization. Whereas the original classical games exhibited inefficient outcomes due to the failure of coordination among players, the quantum games exhibited outcomes in which players were better off due to successive coordination. A deeper investigation could elucidate the details of how the quantization schemes lead to this successful strategic coordination. Further research could also be conducted on the precise mechanisms through which quantization leads to novel results. It was noted in the section on quantum coalitional games that different quantization schemes produce varied results in different games, exemplified by the differences between the quantum version of the "Minority Game" and "Public Goods Game." The current literature does not provide an explanation for this inconsistency in the effects of particular quantization schemes across games. Researching the specific mechanisms that are behind quantization schemes could shed light on why these quantization schemes lead to varied results in different games.
Benjamin, S. C., and Hayden, P. M. (2001). Comment on "Quantum Games and Quantum Strategies.” Physical Review Letters, 87(6), available: doi:10.1103/ physrevlett.87.069801.

Benjamin, S. C., and Hayden, P. M. (2001). Multiplayer quantum games. Physical Review A, 64(3), available: doi:10.1103/physreva.64.030301.

Brassard, G., Broadbent, A., and Tapp, A. (2004). Recasting Mermin's multi-player game into the framework of pseudo-telepathy. arXiv preprint quantph/0408052.

Brassard, G., Broadbent, A., and Tapp, A. (2005). Quantum Pseudo-Telepathy. Foundations of Physics, 35(11), 1877-1907, available: doi:10.1007/s10701005-7353-4.

Chen, K. Y., Hogg, T., and Beausoleil, R. (2002). A quantum treatment of public goods economics. Quantum Information Processing, 1(6), 449-469, available: doi:10.1023/A:1024070415465.

Du, J., Li, H., Xu, X., Shi, M., Wu, J., Zhou, X., and Han, R. (2002). Experimental Realization of Quantum Games on a Quantum Computer. Physical Review Letters, 88(13), available: doi:10.1103/physrevlett.88.137902.

Du, J., Xu, X., Li, H., Shi, M., Zhou, X., and Han, R. (2000). Quantum strategy without entanglement. arXiv preprint quant-ph/0011078.

Du, J., Xu, X., Li, H., Zhou, X., and Han, R. (2000). Nash equilibrium in the quantum battle of sexes game. arXiv preprint quant-ph/0010050.

Eisert, J., Wilkens, M., and Lewenstein, M. (1999). Quantum Games and Quantum Strategies. Physical Review Letters, 83(15), 3077-3080, available: doi:10.1103/physrevlett.83.3077.

Enk, S. J., and Pike, R. (2002). Classical Rules in Quantum Games. Physical Review A, 66(2), available: doi:10.1103/physreva.66.024306

Flitney, A. P., and Hollenberg, L. C. (2008). Quantum minority game utilizing various forms of entanglement. Complex Systems II, 6802, available: doi: $10.1117 / 12.774090$

Grabbe, J. O. (2005). An introduction to quantum game theory. arXiv preprint quant-ph/0506219.

Iqbal, A., and Toor, A. H. (2002). Backwards-induction outcome in a quantum game. Physical Review A, 65(5), available: doi:10.1103/physreva.65.052328.

Li, H., Du, J., and Massar, S. (2002). Continuous-variable quantum games. Physics Letters A, 306(2-3), 73-78, available: doi:10.1016/s0375-9601(02)01628-6.

Marinatto, L., and Weber, T. (2000). A Quantum Approach To Static Games Of Complete Information. Physics Letters A, 272(5-6), 291-303, available: doi:10.1016/s0375-9601(00)00441-2.

Mermin, N. D. (1990). Extreme quantum entanglement in a superposition of macroscopically distinct states. Physical Review Letters, 65(15), 1838-1840, available: doi:10.1103/physrevlett.65.1838.

Meyer, D. A. (1999). Quantum Strategies. Physical Review Letters, 82(5), 10521055, available: doi:10.1103/physrevlett.82.1052.

Nash, J. (1951). Non-Cooperative Games. The Annals of Mathematics, 54(2), 286295, available: doi:10.2307/1969529.

Nielsen, M. A., and Chuang, I. L. (2000). Quantum Computation and Quantum Information. Cambridge: Cambridge University Press.

Prevedel, R., Stefanov, A., Walther, P., and Zeilinger, A. (2007). Experimental realization of a quantum game on a one-way quantum computer. New Journal of Physics, 9(6), 205-205, available: doi:10.1088/1367-2630/9/6/205

Schmid, C., Flitney, A. P., Wieczorek, W., Kiesel, N., Weinfurter, H., and Hollen berg, L. C. (2010). Experimental implementation of a four-player quantum game. New Journal of Physics, 12(6), available: doi:10.1088/13672630/12/6/063031

von Neumann, J. (1955). Mathematical Foundations of Quantum Mechanics. Princeton: Princeton University Press.

von Neumann, J., and Morgenstern, O. (1953). Theory of Games and Economic Behavior. Princeton: Princeton University Press.

\section{REFERENCES}

Benjamin, S. C. (2000). Comment on "A quantum approach to static games of complete information". Physics Letters A, 277(3), 180-182, available: doi:10.1016/s0375-9601(00)00710-6. 